\title{
Strong convergence of a hybrid method for pseudomonotone variational inequalities and fixed point problems
}

\author{
Xin Yu, Yonghong Yao and Yeong-Cheng Liou
}

\begin{abstract}
In this paper, we suggest a hybrid method for finding a common element of the set of solution of a pseudomonotone, Lipschitz-continuous variational inequality problem and the set of common fixed points of an infinite family of nonexpansive mappings. The proposed iterative method combines two well-known methods: extragradient method and $C Q$ method. We derive a necessary and sufficient condition for the strong convergence of the sequences generated by the proposed method.
\end{abstract}

\section{Introduction}

Let $H$ be a real Hilbert space with inner product $\langle\cdot, \cdot\rangle$ and induced norm $\|\cdot\|$. Let $C$ be a nonempty closed convex subset of $H$. Let $A: C \rightarrow H$ be a nonlinear operator. By definition, the variational inequality problem $\operatorname{VI}(C, A)$ is to find $u \in C$ such that

$$
(\mathrm{VI}(\mathrm{C}, \mathrm{A})): \quad\langle A u, v-u\rangle \geq 0, \quad \forall v \in C .
$$

The set of solutions of the variational inequality is denoted by $\Omega$.

Variational inequality theory has emerged as an important tool in studying a wide class of obstacle, unilateral and equilibrium problems, which arise in

Key Words: Variational inequality problem; Fixed point problems; Pseudomonotone mapping; Nonexpansive mapping; Extragradient method; $C Q$ method; Projection.

2010 Mathematics Subject Classification: 47H05; 47H09; 47H10; 47J05; 47J25.

Received: March, 2011.

Revised: April, 2011.

Accepted: February, 2012. 
several branches of pure and applied sciences in a unified and general framework. Several numerical methods have been developed for solving variational inequalities and related optimization problems, see [1], [8], [9],[11]-[14],[21][24], [28]-[31] and the references therein. Let us start with Korpelevich's extragradient method which was introduced by Korpelevich [13] in 1976 and which generates a sequence $\left\{x_{n}\right\}$ via the recursion:

$$
\left\{\begin{array}{l}
y_{n}=P_{C}\left[x_{n}-\lambda A x_{n}\right], \\
x_{n+1}=P_{C}\left[x_{n}-\lambda A y_{n}\right], n \geq 0,
\end{array}\right.
$$

where $P_{C}$ is the metric projection from $R^{n}$ onto $C, A: C \rightarrow H$ is a monotone operator and $\lambda$ is a constant. Korpelevich [13] proved that the sequence $\left\{x_{n}\right\}$ converges strongly to a solution of $\operatorname{VI}(C, A)$. Note that the setting of the problem is the Euclidean space $R^{n}$.

Korpelevich's extragradient method has extensively been studied in the literature for solving a more general problem that consists of finding a common point that lies in the solution set of a variational inequality and the set of fixed points of a nonexpansive mapping. This type of problem aries in various theoretical and modeling contexts, see e.g., [2],[4]-[7],[15],[25],[26] and references therein. Especially, Nadezhkina and Takahashi [17] introduced the following iterative method which combines Korpelevich's extragradient method and a $C Q$ method:

$$
\begin{aligned}
& x_{0}=x \in C, \\
& y_{n}=P_{C}\left[x_{n}-\lambda_{n} A x_{n}\right], \\
& z_{n}=\alpha_{n} x_{n}+\left(1-\alpha_{n}\right) S P_{C}\left[x_{n}-\lambda_{n} A y_{n}\right], \\
& C_{n}=\left\{z \in C:\left\|z_{n}-z\right\| \leq\left\|x_{n}-z\right\|\right\}, \\
& Q_{n}=\left\{z \in C:\left\langle x_{n}-z, x-x_{n}\right\rangle \geq 0\right\}, \\
& x_{n+1}=P_{C_{n} \cap Q_{n}} x, n \geq 0, n \geq 0,
\end{aligned}
$$

where $P_{C}$ is the metric projection from $H$ onto $C, A: C \rightarrow H$ is a monotone $k$-Lipschitz-continuous mapping, $S: C \rightarrow C$ is a nonexpansive mapping, $\left\{\lambda_{n}\right\}$ and $\left\{\alpha_{n}\right\}$ are two real number sequences. They proved the strong convergence of the sequences $\left\{x_{n}\right\},\left\{y_{n}\right\}$ and $\left\{z_{n}\right\}$ to the same element in $F i x(S) \cap \Omega$. We note that Nadezhkina and Takahashi [17] employed the monotonicity and Lipschitz-continuity of $A$ to define a maximal monotone operator $T$ as follows:

$$
T v=\left\{\begin{aligned}
A v+N_{C} v, & \text { if } v \in C, \\
\emptyset, & \text { if } v \notin C .
\end{aligned}\right.
$$

where $N_{C} v=\{w \in H:\langle v-u, w\rangle \geq 0, \forall u \in C\}$ is the normal cone to $C$ at $v \in C$ (see, [19]). However, if the mapping $A$ is a pseudomonotone Lipschitz- 
continuous, then $T$ is not necessarily a maximal monotone operator. This fact implies that the approach used in [17] cannot be applied. To overcome this difficulty, Ceng, Teboulle and Yao [3] suggested a new iterative method as follows:

$$
\begin{aligned}
& y_{n}=P_{C}\left[x_{n}-\lambda_{n} A x_{n}\right], \\
& z_{n}=\alpha_{n} x_{n}+\left(1-\alpha_{n}\right) S_{n} P_{C}\left[x_{n}-\lambda_{n} A y_{n}\right], \\
& C_{n}=\left\{z \in C:\left\|z_{n}-z\right\| \leq\left\|x_{n}-z\right\|\right\}, \\
& \text { find } x_{n+1} \in C_{n} \text { such that } \\
& \left\langle x_{n}-x_{n+1}+e_{n}-\sigma_{n} A x_{n+1}, x_{n+1}-x\right\rangle \geq-\epsilon_{n}, \quad \forall x \in C_{n},
\end{aligned}
$$

where $A: C \rightarrow H$ is a pseudomonotone, $k$-lipschitz-continuous and $(w, s)$ sequentially-continuous mapping, $\left\{S_{i}\right\}_{i=1}^{N}: C \rightarrow C$ are $N$ nonexpansive mappings. Under some mild conditions, they proved that the sequences $\left\{x_{n}\right\},\left\{y_{n}\right\}$ and $\left\{z_{n}\right\}$ converge weakly to the same element of $\bigcap_{i=1}^{N} F i x\left(S_{i}\right) \cap \Omega$ if and only if $\liminf \inf _{n}\left\langle A x_{n}, x-x_{n}\right\rangle \geq 0, \forall x \in C$. Note that Ceng, Teboulle and Yao's method has only weak convergence. So, we may ask of whether $i$ ) a strong convergence property is available, $i i)$ a denumerable family of maps $\left(S_{i} ; i \geq 1\right)$ is allowed.

Motivated and inspired by the works of Nadezhkina and Takahashi [17] and Ceng, Teboulle and Yao [3], in this paper we suggest a hybrid method for finding a common element of the set of solution of a pseudomonotone, Lipschitz-continuous variational inequality problem and the set of common fixed points of an infinite family of nonexpansive mappings. The proposed iterative method combines two well-known methods: extragradient method and $C Q$ method. We derive a necessary and sufficient condition for the strong convergence of the sequences generated by the proposed method.

\section{Preliminaries}

In this section, we will recall some basic notations and collect some conclusions that will be used in the next section.

Let $C$ be a nonempty closed convex subset of a real Hilbert space $H$. A mapping $A: C \rightarrow H$ is called monotone if

$$
\langle A u-A v, u-v\rangle \geq 0, \forall u, v \in C .
$$

A mapping $A: C \rightarrow H$ is called pseudomonotone if, for all $u, v \in C$,

$$
\langle A u, v-u \geq 0 \Rightarrow\langle A v, v-u\rangle \geq 0 .
$$

It is clear that if a mapping $A$ is monotone, then it is pseudomonotone. 
Recall that a mapping $S: C \rightarrow C$ is said to be nonexpansive if

$$
\|S x-S y\| \leq\|x-y\|, \forall x, y \in C .
$$

Denote by $F i x(S)$ the set of fixed points of $S$; that is, Fix $(S)=\{x \in C$ : $S x=x\}$.

It is well known that, for any $u \in H$, there exists a unique $u_{0} \in C$ such that

$$
\left\|u-u_{0}\right\|=\inf \{\|u-x\|: x \in C\} .
$$

We denote $u_{0}$ by $P_{C}[u]$, where $P_{C}$ is called the metric projection of $H$ onto $C$. The metric projection $P_{C}$ of $H$ onto $C$ has the following basic properties:

(i) $\left\|P_{C}[x]-P_{C}[y]\right\| \leq\|x-y\|$ for all $x, y \in H$.

(ii) $\left\langle x-P_{C}[x], y-P_{C}[x]\right\rangle \leq 0$ for all $x \in H, y \in C$.

(iii) The property (ii) is equivalent to

$$
\left\|x-P_{C}[x]\right\|^{2}+\left\|y-P_{C}[x]\right\|^{2} \leq\|x-y\|, \forall x \in H, y \in C .
$$

(iv) In the context of the variational inequality problem, the characterization of the projection implies that

$$
u \in \Omega \Leftrightarrow u=P_{C}[u-\lambda A u], \forall \lambda>0
$$

Recall that $H$ satisfies the Opial condition [27]; i.e., for any sequence $\left\{x_{n}\right\}$ with $x_{n}$ converges weakly to $x$, the inequality

$$
\liminf _{n \rightarrow \infty}\left\|x_{n}-x\right\|<\liminf _{n \rightarrow \infty}\left\|x_{n}-y\right\|
$$

holds for every $y \in H$ with $y \neq x$.

Let $C$ be a nonempty closed convex subset of a real Hilbert space $H$. Let $\left\{S_{i}\right\}_{i=1}^{\infty}$ be infinite family of nonexpansive mappings of $C$ into itself and let $\left\{\xi_{i}\right\}_{i=1}^{\infty}$ be real number sequences such that $0 \leq \xi_{i} \leq 1$ for every $i \in \mathbf{N}$. For 
any $n \in \mathbf{N}$, define a mapping $W_{n}$ of $C$ into itself as follows:

$$
\begin{aligned}
& U_{n, n+1}=I, \\
& U_{n, n}=\xi_{n} S_{n} U_{n, n+1}+\left(1-\xi_{n}\right) I, \\
& U_{n, n-1}=\xi_{n-1} S_{n-1} U_{n, n}+\left(1-\xi_{n-1}\right) I, \\
& \quad \vdots \\
& U_{n, k}=\xi_{k} S_{k} U_{n, k+1}+\left(1-\xi_{k}\right) I, \\
& U_{n, k-1}=\xi_{k-1} S_{k-1} U_{n, k}+\left(1-\xi_{k-1}\right) I, \\
& \quad \vdots \\
& U_{n, 2}=\xi_{2} S_{2} U_{n, 3}+\left(1-\xi_{2}\right) I, \\
& W_{n}=U_{n, 1}=\xi_{1} S_{1} U_{n, 2}+\left(1-\xi_{1}\right) I .
\end{aligned}
$$

Such $W_{n}$ is called the $W$-mapping generated by $\left\{S_{i}\right\}_{i=1}^{\infty}$ and $\left\{\xi_{i}\right\}_{i=1}^{\infty}$.

We have the following crucial Lemmas 3.1 and 3.2 concerning $W_{n}$ which can be found in [20]. Now we only need the following similar version in Hilbert spaces.

Lemma 2.1. Let $C$ be a nonempty closed convex subset of a real Hilbert space $H$. Let $S_{1}, S_{2}, \cdots$ be nonexpansive mappings of $C$ into itself such that $\bigcap_{n=1}^{\infty} \operatorname{Fix}\left(S_{n}\right)$ is nonempty, and let $\xi_{1}, \xi_{2}, \cdots$ be real numbers such that $0<$ $\xi_{i} \leq b<1$ for any $i \in \mathbf{N}$. Then, for every $x \in C$ and $k \in \mathbf{N}$, the limit $\lim _{n \rightarrow \infty} U_{n, k} x$ exists.

Lemma 2.2. Let $C$ be a nonempty closed convex subset of a real Hilbert space $H$. Let $S_{1}, S_{2}, \cdots$ be nonexpansive mappings of $C$ into itself such that $\bigcap_{n=1}^{\infty} F i x\left(S_{n}\right)$ is nonempty, and let $\xi_{1}, \xi_{2}, \cdots$ be real numbers such that $0<$ $\xi_{i} \leq b<1$ for any $i \in N$. Then, $\operatorname{Fix}(W)=\bigcap_{n=1}^{\infty} \operatorname{Fix}\left(S_{n}\right)$.

Lemma 2.3. (see [27]) Using Lemmas 2.1 and 2.2, one can define a mapping $W$ of $C$ into itself as: $W x=\lim _{n \rightarrow \infty} W_{n} x=\lim _{n \rightarrow \infty} U_{n, 1} x$, for every $x \in C$. If $\left\{x_{n}\right\}$ is a bounded sequence in $C$, then we have

$$
\lim _{n \rightarrow \infty}\left\|W x_{n}-W_{n} x_{n}\right\|=0
$$

We also need the following well-known lemmas for proving our main results.

Lemma 2.4. ([10]) Let $C$ be a nonempty closed convex subset of a real Hilbert space $H$. Let $S: C \rightarrow C$ be a nonexpansive mapping with $F i x(S) \neq \emptyset$. Then $S$ is demiclosed on $C$, i.e., if $y_{n} \rightarrow z \in C$ weakly and $y_{n}-S y_{n} \rightarrow y$ strongly, then $(I-S) z=y$. 
Lemma 2.5. ([16]) Let $C$ be a closed convex subset of $H$. Let $\left\{x_{n}\right\}$ be a sequence in $H$ and $u \in H$. Let $q=P_{C}[u]$. If $\left\{x_{n}\right\}$ is such that $\omega_{w}\left(x_{n}\right) \subset C$ and satisfies the condition

$$
\left\|x_{n}-u\right\| \leq\|u-q\| \text { for all } n \text {. }
$$

Then $x_{n} \rightarrow q$.

We adopt the following notation:

- For a given sequence $\left\{x_{n}\right\} \subset H, \omega_{w}\left(x_{n}\right)$ denotes the weak $\omega$-limit set of $\left\{x_{n}\right\}$; that is, $\omega_{w}\left(x_{n}\right):=\left\{x \in H:\left\{x_{n_{j}}\right\}\right.$ converges weakly to $x$ for some subsequence $\left\{n_{j}\right\}$ of $\left.\{n\}\right\}$.

- $x_{n} \rightarrow x$ stands for the weak convergence of $\left(x_{n}\right)$ to $x$;

- $x_{n} \rightarrow x$ stands for the strong convergence of $\left(x_{n}\right)$ to $x$.

\section{Main results}

In this section we will state and prove our main results.

Theorem 3.1. Let $C$ be a nonempty closed convex subset of a real Hilbert space $H$. Let $A: C \rightarrow H$ be a pseudomonotone, $k$-Lipschitz-continuous and $(w, s)$-sequentially-continuous mapping and let $\left\{S_{n}\right\}_{n=1}^{\infty}$ be an infinite family of nonexpansive mappings of $C$ into itself such that $\bigcap_{n=1}^{\infty} F i x\left(S_{n}\right) \cap \Omega \neq \emptyset$. Let $x_{1}=x_{0} \in C$. For $x_{1} \in C, C_{1}=C$, let $\left\{x_{n}\right\},\left\{y_{n}\right\},\left\{z_{n}\right\}$ and $\left\{C_{n}\right\}$ be sequences generated as:

$$
\begin{aligned}
& y_{n}=P_{C_{n}}\left[x_{n}-\lambda_{n} A x_{n}\right], \\
& z_{n}=\alpha_{n} x_{n}+\left(1-\alpha_{n}\right) W_{n} P_{C_{n}}\left[x_{n}-\lambda_{n} A y_{n}\right], \\
& C_{n+1}=\left\{z \in C_{n}:\left\|z_{n}-z\right\| \leq\left\|x_{n}-z\right\|\right\}, \\
& x_{n+1}=P_{C_{n+1}}\left[x_{0}\right], n \geq 1,
\end{aligned}
$$

where $\left\{W_{n} ; n \geq 1\right\}$ are $W$-mappings of (2). Assume that

(i) $\left\{\lambda_{n}\right\} \subset[a, b]$ for some $a, b \in(0,1 / k)$;

(ii) $\left\{\alpha_{n}\right\} \subset[0, c]$ for some $c \in[0,1)$.

Then the sequences $\left\{x_{n}\right\},\left\{y_{n}\right\}$ and $\left\{z_{n}\right\}$ generated by (3) converge strongly to the same point $P_{\bigcap_{n=1}^{\infty}} \operatorname{Fix}\left(S_{n}\right) \cap \Omega\left[x_{0}\right]$ if and only if $\liminf _{n}\left\langle A x_{n}, x-x_{n}\right\rangle \geq 0$, $\forall x \in C$. 
The proof will be divided into several conclusions. Assume in the sequel that all assumptions of Theorem 3.1 are satisfied.

Conclusion 3.2. (1) Every $C_{n}$ is closed and convex, $n \geq 1$;

(2) $\bigcap_{n=1}^{\infty} \operatorname{Fix}\left(S_{n}\right) \cap \Omega \subset C_{n+1}, \forall n \geq 1$;

(3) $\left\{x_{n+1}\right\}$ is well-defined.

Proof. First we note that $C_{1}=C$ is closed and convex. Assume that $C_{k}$ is closed and convex. From (3), we can rewrite $C_{k+1}$ as

$$
C_{k+1}=\left\{z \in C_{k}:\left\langle z-\frac{x_{k}+z_{k}}{2}, z_{k}-x_{k}\right\rangle \geq 0\right\} .
$$

It is clear that $C_{k+1}$ is a half space. Hence, $C_{k+1}$ is closed and convex. By induction, we deduce that $C_{n}$ is closed and convex for all $n \geq 1$. Next we show that $\bigcap_{n=1}^{\infty} \operatorname{Fix}\left(S_{n}\right) \cap \Omega \subset C_{n+1}, \forall n \geq 1$.

Set $t_{n}=P_{C_{n}}\left[x_{n}-\lambda A y_{n}\right]$ for all $n \geq 1$. Pick up $u \in \bigcap_{n=1}^{\infty} \operatorname{Fix}\left(S_{n}\right) \cap \Omega$. From property (iii) of $P_{C}$, we have

$$
\begin{aligned}
\left\|t_{n}-u\right\|^{2} & \leq\left\|x_{n}-\lambda_{n} A y_{n}-u\right\|^{2}-\left\|x_{n}-\lambda_{n} A y_{n}-t_{n}\right\|^{2} \\
& =\left\|x_{n}-u\right\|^{2}-\left\|x_{n}-t_{n}\right\|^{2}+2 \lambda_{n}\left\langle A y_{n}, u-t_{n}\right\rangle \\
& =\left\|x_{n}-u\right\|^{2}-\left\|x_{n}-t_{n}\right\|^{2}+2 \lambda_{n}\left\langle A y_{n}, u-y_{n}\right\rangle+2 \lambda_{n}\left\langle A y_{n}, y_{n}-t_{\left(n_{n}(4)\right.}\right)
\end{aligned}
$$

Since $u \in \Omega$ and $y_{n} \in C_{n} \subset C$, we get

$$
\left\langle A u, y_{n}-u\right\rangle \geq 0
$$

This together with the pseudomonotonicity of $A$ imply that

$$
\left\langle A y_{n}, y_{n}-u\right\rangle \geq 0 \text {. }
$$

Combine (4) with (5) to deduce

$$
\begin{aligned}
\left\|t_{n}-u\right\|^{2} \leq & \left\|x_{n}-u\right\|^{2}-\left\|x_{n}-t_{n}\right\|^{2}+2 \lambda_{n}\left\langle A y_{n}, y_{n}-t_{n}\right\rangle \\
= & \left\|x_{n}-u\right\|^{2}-\left\|x_{n}-y_{n}\right\|-2\left\langle x_{n}-y_{n}, y_{n}-t_{n}\right\rangle-\left\|y_{n}-t_{n}\right\|^{2} \\
& +2 \lambda_{n}\left\langle A y_{n}, y_{n}-t_{n}\right\rangle \\
= & \left\|x_{n}-u\right\|^{2}-\left\|x_{n}-y_{n}\right\|^{2}-\left\|y_{n}-t_{n}\right\|^{2} \\
& +2\left\langle x_{n}-\lambda_{n} A y_{n}-y_{n}, t_{n}-y_{n}\right\rangle .
\end{aligned}
$$

Note that $y_{n}=P_{C_{n}}\left[x_{n}-\lambda_{n} A x_{n}\right]$ and $t_{n} \in C_{n}$. Then, by using the property (ii) of $P_{C}$, we have

$$
\left\langle x_{n}-\lambda_{n} A x_{n}-y_{n}, t_{n}-y_{n}\right\rangle \leq 0 \text {. }
$$


Hence,

$$
\begin{aligned}
\left\langle x_{n}-\lambda_{n} A y_{n}-y_{n}, t_{n}-y_{n}\right\rangle & =\left\langle x_{n}-\lambda_{n} A x_{n}-y_{n}, t_{n}-y_{n}\right\rangle+\left\langle\lambda_{n} A x_{n}-\lambda_{n} A y_{n}, t_{n}-y_{n}\right\rangle \\
& \leq\left\langle\lambda_{n} A x_{n}-\lambda_{n} A y_{n}, t_{n}-y_{n}\right\rangle \\
& \leq \lambda_{n} k\left\|x_{n}-y_{n}\right\|\left\|t_{n}-y_{n}\right\| .
\end{aligned}
$$

From (6) and (7), we get

$$
\begin{aligned}
\left\|t_{n}-u\right\|^{2} & \leq\left\|x_{n}-u\right\|^{2}-\left\|x_{n}-y_{n}\right\|^{2}-\left\|y_{n}-t_{n}\right\|^{2}+2 \lambda_{n} k\left\|x_{n}-y_{n}\right\|\left\|t_{n}-y_{n}\right\| \\
& \leq\left\|x_{n}-u\right\|^{2}-\left\|x_{n}-y_{n}\right\|^{2}-\left\|y_{n}-t_{n}\right\|^{2}+\lambda_{n}^{2} k^{2}\left\|x_{n}-y_{n}\right\|^{2}+\left\|y_{n}-t_{n}\right\|^{2} \\
& =\left\|x_{n}-u\right\|^{2}+\left(\lambda_{n}^{2} k^{2}-1\right)\left\|x_{n}-y_{n}\right\|^{2} \\
& \leq\left\|x_{n}-u\right\|^{2} .
\end{aligned}
$$

Therefore, from (8), together with $z_{n}=\alpha_{n} x_{n}+\left(1-\alpha_{n}\right) W_{n} t_{n}$ and $u=W_{n} u$, we get

$$
\begin{aligned}
\left\|z_{n}-u\right\|^{2} & =\left\|\alpha_{n}\left(x_{n}-u\right)+\left(1-\alpha_{n}\right)\left(W_{n} t_{n}-u\right)\right\|^{2} \\
& \leq \alpha_{n}\left\|x_{n}-u\right\|^{2}+\left(1-\alpha_{n}\right)\left\|W_{n} t_{n}-u\right\|^{2} \\
& \leq \alpha_{n}\left\|x_{n}-u\right\|^{2}+\left(1-\alpha_{n}\right)\left\|t_{n}-u\right\|^{2} \\
& \leq\left\|x_{n}-u\right\|^{2}+\left(1-\alpha_{n}\right)\left(\lambda_{n}^{2} k^{2}-1\right)\left\|x_{n}-y_{n}\right\|^{2} \\
& \leq\left\|x_{n}-u\right\|^{2}
\end{aligned}
$$

which implies that

$$
u \in C_{n+1} .
$$

Therefore,

$$
\bigcap_{n=1}^{\infty} F i x\left(S_{n}\right) \cap \Omega \subset C_{n+1}, \forall n \geq 1 .
$$

This implies that $\left\{x_{n+1}\right\}$ is well-defined.

Conclusion 3.3. The sequences $\left\{x_{n}\right\},\left\{z_{n}\right\}$ and $\left\{t_{n}\right\}$ are all bounded and $\lim _{n \rightarrow \infty}\left\|x_{n}-x_{0}\right\|$ exists.

Proof. From $x_{n+1}=P_{C_{n+1}}\left[x_{0}\right]$, we have

$$
\left\langle x_{0}-x_{n+1}, x_{n+1}-y\right\rangle \geq 0, \forall y \in C_{n+1} .
$$

Since $\bigcap_{n=1}^{\infty} F i x\left(S_{n}\right) \cap \Omega \subset C_{n+1}$, we also have

$$
\left\langle x_{0}-x_{n+1}, x_{n+1}-u\right\rangle \geq 0, \forall u \in \bigcap_{n=1}^{\infty} \operatorname{Fix}\left(S_{n}\right) \cap \Omega .
$$


So, for $u \in \bigcap_{n=1}^{\infty} \operatorname{Fix}\left(S_{n}\right) \cap \Omega$, we have

$$
\begin{aligned}
0 & \leq\left\langle x_{0}-x_{n+1}, x_{n+1}-u\right\rangle \\
& =\left\langle x_{0}-x_{n+1}, x_{n+1}-x_{0}+x_{0}-u\right\rangle \\
& =-\left\|x_{0}-x_{n+1}\right\|^{2}+\left\langle x_{0}-x_{n+1}, x_{0}-u\right\rangle \\
& \leq-\left\|x_{0}-x_{n+1}\right\|^{2}+\left\|x_{0}-x_{n+1}\right\|\left\|x_{0}-u\right\| .
\end{aligned}
$$

Hence,

$$
\left\|x_{0}-x_{n+1}\right\| \leq\left\|x_{0}-u\right\|, \forall u \in \bigcap_{n=1}^{\infty} F i x\left(S_{n}\right) \cap \Omega,
$$

which implies that $\left\{x_{n}\right\}$ is bounded. From (8) and (9), we can deduce that $\left\{z_{n}\right\}$ and $\left\{t_{n}\right\}$ are also bounded.

From $x_{n}=P_{C_{n}}\left[x_{0}\right]$ and $x_{n+1}=P_{C_{n+1}}\left[x_{0}\right] \in C_{n+1} \subset C_{n}$, we have

$$
\left\langle x_{0}-x_{n}, x_{n}-x_{n+1}\right\rangle \geq 0 \text {. }
$$

Hence,

$$
\begin{aligned}
0 & \leq\left\langle x_{0}-x_{n}, x_{n}-x_{n+1}\right\rangle \\
& =\left\langle x_{0}-x_{n}, x_{n}-x_{0}+x_{0}-x_{n+1}\right\rangle \\
& =-\left\|x_{0}-x_{n}\right\|^{2}+\left\langle x_{0}-x_{n}, x_{0}-x_{n+1}\right\rangle \\
& \leq-\left\|x_{0}-x_{n}\right\|^{2}+\left\|x_{0}-x_{n}\right\|\left\|x_{0}-x_{n+1}\right\|,
\end{aligned}
$$

and therefore

$$
\left\|x_{0}-x_{n}\right\| \leq\left\|x_{0}-x_{n+1}\right\| .
$$

This together with the boundedness of the sequence $\left\{x_{n}\right\}$ imply that $\lim _{n \rightarrow \infty} \| x_{n}-$ $x_{0} \|$ exists.

Conclusion 3.4. $\lim _{n \rightarrow \infty}\left\|x_{n+1}-x_{n}\right\|=\lim _{n \rightarrow \infty}\left\|x_{n}-y_{n}\right\|=\lim _{n \rightarrow \infty} \| x_{n}-$ $z_{n}\left\|=\lim _{n \rightarrow \infty}\right\| x_{n}-t_{n} \|=0$ and $\lim _{n \rightarrow \infty}\left\|x_{n}-W_{n} x_{n}\right\|=\lim _{n \rightarrow \infty} \| x_{n}-$ $W x_{n} \|=0$.

Proof. It is well-known that in Hilbert spaces $H$, the following identity holds:

$$
\|x-y\|^{2}=\|x\|^{2}-\|y\|^{2}-2\langle x-y, y\rangle, \quad \forall x, y \in H .
$$

Therefore,

$$
\begin{aligned}
\left\|x_{n+1}-x_{n}\right\|^{2} & =\left\|\left(x_{n+1}-x_{0}\right)-\left(x_{n}-x_{0}\right)\right\|^{2} \\
& =\left\|x_{n+1}-x_{0}\right\|^{2}-\left\|x_{n}-x_{0}\right\|^{2}-2\left\langle x_{n+1}-x_{n}, x_{n}-x_{0}\right\rangle .
\end{aligned}
$$


It follows from (11) that

$$
\left\|x_{n+1}-x_{n}\right\|^{2} \leq\left\|x_{n+1}-x_{0}\right\|^{2}-\left\|x_{n}-x_{0}\right\|^{2} .
$$

Since $\lim _{n \rightarrow \infty}\left\|x_{n}-x_{0}\right\|$ exists, we get $\left\|x_{n+1}-x_{0}\right\|^{2}-\left\|x_{n}-x_{0}\right\|^{2} \rightarrow 0$. Therefore,

$$
\lim _{n \rightarrow \infty}\left\|x_{n+1}-x_{n}\right\|=0
$$

Since $x_{n+1} \in C_{n}$, we have

$$
\left\|z_{n}-x_{n+1}\right\| \leq\left\|x_{n}-x_{n+1}\right\|
$$

and hence

$$
\begin{aligned}
\left\|x_{n}-z_{n}\right\| & \leq\left\|x_{n}-x_{n+1}\right\|+\left\|x_{n+1}-z_{n}\right\| \\
& \leq 2\left\|x_{n+1}-x_{n}\right\| \\
& \rightarrow 0 .
\end{aligned}
$$

For each $u \in \bigcap_{n=1}^{\infty} \operatorname{Fix}\left(S_{n}\right) \cap \Omega$, from (9), we have

$$
\begin{aligned}
\left\|x_{n}-y_{n}\right\|^{2} & \leq \frac{1}{\left(1-\alpha_{n}\right)\left(1-\lambda_{n}^{2} k^{2}\right)}\left(\left\|x_{n}-u\right\|^{2}-\left\|z_{n}-u\right\|^{2}\right) \\
& \leq \frac{1}{\left(1-\alpha_{n}\right)\left(1-\lambda_{n}^{2} k^{2}\right)}\left(\left\|x_{n}-u\right\|+\left\|z_{n}-u\right\|\right)\left\|x_{n}-z_{n}\right\| .
\end{aligned}
$$

Since $\left\|x_{n}-z_{n}\right\| \rightarrow 0$ and the sequences $\left\{x_{n}\right\}$ and $\left\{z_{n}\right\}$ are bounded, we obtain $\left\|x_{n}-y_{n}\right\| \rightarrow 0$.

We note that

$$
\begin{aligned}
\left\|t_{n}-u\right\|^{2} & \leq\left\|x_{n}-u\right\|^{2}-\left\|x_{n}-y_{n}\right\|^{2}-\left\|y_{n}-t_{n}\right\|^{2}+2 \lambda_{n} k\left\|x_{n}-y_{n}\right\|\left\|t_{n}-y_{n}\right\| \\
& \leq\left\|x_{n}-u\right\|^{2}-\left\|x_{n}-y_{n}\right\|^{2}-\left\|y_{n}-t_{n}\right\|^{2}+\left\|x_{n}-y_{n}\right\|^{2}+\lambda_{n}^{2} k^{2}\left\|y_{n}-t_{n}\right\|^{2} \\
& =\left\|x_{n}-u\right\|^{2}+\left(\lambda_{n}^{2} k^{2}-1\right)\left\|y_{n}-t_{n}\right\|^{2} .
\end{aligned}
$$

Hence,

$$
\begin{aligned}
\left\|z_{n}-u\right\|^{2} & \leq \alpha_{n}\left\|x_{n}-u\right\|^{2}+\left(1-\alpha_{n}\right)\left\|t_{n}-u\right\|^{2} \\
& \leq \alpha_{n}\left\|x_{n}-u\right\|^{2}+\left(1-\alpha_{n}\right)\left(\left\|x_{n}-u\right\|^{2}+\left(\lambda_{n}^{2} k^{2}-1\right)\left\|y_{n}-t_{n}\right\|^{2}\right) \\
& =\left\|x_{n}-u\right\|^{2}+\left(1-\alpha_{n}\right)\left(\lambda_{n}^{2} k^{2}-1\right)\left\|y_{n}-t_{n}\right\|^{2} .
\end{aligned}
$$

It follows that

$$
\begin{aligned}
\left\|t_{n}-y_{n}\right\|^{2} & \leq \frac{1}{\left(1-\alpha_{n}\right)\left(1-\lambda_{n}^{2} k^{2}\right)}\left(\left\|x_{n}-u\right\|^{2}-\left\|z_{n}-u\right\|^{2}\right) \\
& \leq \frac{1}{\left(1-\alpha_{n}\right)\left(1-\lambda_{n}^{2} k^{2}\right)}\left(\left\|x_{n}-u\right\|+\left\|z_{n}-u\right\|\right)\left\|x_{n}-z_{n}\right\| \\
& \rightarrow 0 .
\end{aligned}
$$


Since $A$ is $k$-Lipschitz-continuous, we have $\left\|A y_{n}-A t_{n}\right\| \rightarrow 0$. From

$$
\left\|x_{n}-t_{n}\right\| \leq\left\|x_{n}-y_{n}\right\|+\left\|y_{n}-t_{n}\right\|,
$$

we also have

$$
\left\|x_{n}-t_{n}\right\| \rightarrow 0
$$

Since $z_{n}=\alpha_{n} x_{n}+\left(1-\alpha_{n}\right) W_{n} t_{n}$, we have

$$
\left(1-\alpha_{n}\right)\left(W_{n} t_{n}-t_{n}\right)=\alpha_{n}\left(t_{n}-x_{n}\right)+\left(z_{n}-t_{n}\right) .
$$

Then,

$$
\begin{aligned}
(1-c)\left\|W_{n} t_{n}-t_{n}\right\| & \leq\left(1-\alpha_{n}\right)\left\|W_{n} t_{n}-t_{n}\right\| \\
& \leq \alpha_{n}\left\|t_{n}-x_{n}\right\|+\left\|z_{n}-t_{n}\right\| \\
& \leq\left(1+\alpha_{n}\right)\left\|t_{n}-x_{n}\right\|+\left\|z_{n}-x_{n}\right\|
\end{aligned}
$$

and hence $\left\|t_{n}-W_{n} t_{n}\right\| \rightarrow 0$. Observe also that

$$
\begin{aligned}
\left\|x_{n}-W_{n} x_{n}\right\| & \leq\left\|x_{n}-t_{n}\right\|+\left\|t_{n}-W_{n} t_{n}\right\|+\left\|W_{n} t_{n}-W_{n} x_{n}\right\| \\
& \leq\left\|x_{n}-t_{n}\right\|+\left\|t_{n}-W_{n} t_{n}\right\|+\left\|t_{n}-x_{n}\right\| \\
& \leq 2\left\|x_{n}-t_{n}\right\|+\left\|t_{n}-W_{n} t_{n}\right\| .
\end{aligned}
$$

So, we have $\left\|x_{n}-W_{n} x_{n}\right\| \rightarrow 0$. On the other hand, since $\left\{x_{n}\right\}$ is bounded, from Lemma 2.3, we have $\lim _{n \rightarrow \infty}\left\|W_{n} x_{n}-W x_{n}\right\|=0$. Therefore, we have

$$
\lim _{n \rightarrow \infty}\left\|x_{n}-W x_{n}\right\|=0 .
$$

Proof. Proof of Theorem 3.1, continued. First, we prove the necessity. Suppose that $\left\{x_{n}\right\},\left\{y_{n}\right\}$ and $\left\{z_{n}\right\}$ converge strongly to the same element $\tilde{u} \in$ $\bigcap_{n=1}^{\infty} \operatorname{Fix}\left(S_{n}\right) \cap \Omega$. From the $(w, s)$-sequential continuity of $A$, we have $A x_{n} \rightarrow$ $A \tilde{u}$. Observe that, for every $x \in C$,

$$
\begin{aligned}
\left|\left\langle A x_{n}, x-x_{n}\right\rangle-\langle A \tilde{u}, x-\tilde{u}\rangle\right| \leq & \left|\left\langle A x_{n}, x-x_{n}\right\rangle-\left\langle A \tilde{u}, x-x_{n}\right\rangle\right| \\
& +\left|\left\langle A \tilde{u}, x-x_{n}\right\rangle-\langle A \tilde{u}, x-\tilde{u}\rangle\right| \\
= & \left|\left\langle A x_{n}-A \tilde{u}, x-x_{n}\right\rangle\right|+\left|\left\langle A \tilde{u}, \tilde{u}-x_{n}\right\rangle\right| \\
\leq & \left\|A x_{n}-A \tilde{u}\right\|\left\|x-x_{n}\right\|+\left|\left\langle A \tilde{u}, \tilde{u}-x_{n}\right\rangle\right| .
\end{aligned}
$$

This implies that

$$
\liminf _{n \rightarrow \infty}\left\langle A x_{n}, x-x_{n}\right\rangle=\lim _{n \rightarrow \infty}\left\langle A x_{n}, x-x_{n}\right\rangle=\langle A \tilde{u}, x-\tilde{u}\rangle, \forall x \in C .
$$


Consequently, the necessity holds.

Next, we prove the sufficiency. By Conclusions 3.3-3.5, we have

$$
\lim _{n \rightarrow \infty}\left\|x_{n}-W x_{n}\right\|=0
$$

Furthermore, since $\left\{x_{n}\right\}$ is bounded, it has a subsequence $\left\{x_{n_{j}}\right\}$ which converges weakly to some $\tilde{u} \in C$; hence, we have $\lim _{j \rightarrow \infty}\left\|x_{n_{j}}-W x_{n_{j}}\right\|=0$. Note that, from Lemma 2.4, it follows that $I-W$ is demiclosed at zero. Thus $\tilde{u} \in \operatorname{Fix}(W)$. Observe that, for every $x \in C$,

$$
\begin{aligned}
& \left|\left\langle A x_{n_{j}}, x-x_{n_{j}}\right\rangle-\langle A \tilde{u}, x-\tilde{u}\rangle\right| \\
\leq & \left|\left\langle A x_{n_{j}}, x-x_{n_{j}}\right\rangle-\left\langle A \tilde{u}, x-x_{n_{j}}\right\rangle\right|+\left|\left\langle A \tilde{u}, x-x_{n_{j}}\right\rangle-\langle A \tilde{u}, x-\tilde{u}\rangle\right| \\
= & \left|\left\langle A x_{n_{j}}-A \tilde{u}, x-x_{n_{j}}\right\rangle\right|+\left|\left\langle A \tilde{u}, \tilde{u}-x_{n_{j}}\right\rangle\right| \\
\leq & \left\|A x_{n_{j}}-A \tilde{u}\right\|\left\|x-x_{n_{j}}\right\|+\left|\left\langle A \tilde{u}, \tilde{u}-x_{n_{j}}\right\rangle\right| .
\end{aligned}
$$

From the $(w, s)$-sequential continuity of $A$, it follows that $\lim _{j \rightarrow \infty} \| A x_{n_{j}}-$ $A \tilde{u} \|=0$. Hence, we have

$$
\langle A \tilde{u}, x-\tilde{u}\rangle=\lim _{j \rightarrow \infty}\left\langle A x_{n_{j}}, x-x_{n_{j}}\right\rangle \geq \liminf _{n \rightarrow \infty}\left\langle A x_{n}, x-x_{n}\right\rangle \geq 0, \forall x \in C .
$$

This implies that $\tilde{u} \in \Omega$. Consequently, $\tilde{u} \in \bigcap_{n=1}^{\infty} F i x\left(S_{n}\right) \cap \Omega$. That is, $\omega_{w}\left(x_{n}\right) \subset \bigcap_{n=1}^{\infty} \operatorname{Fix}\left(S_{n}\right) \cap \Omega$.

In (10), if we take $u=P_{\bigcap_{n=1}^{\infty} F i x\left(S_{n}\right) \cap \Omega}\left[x_{0}\right]$, we get

$$
\left\|x_{0}-x_{n+1}\right\| \leq\left\|x_{0}-P_{\bigcap_{n=1}^{\infty} \operatorname{Fix}\left(S_{n}\right) \cap \Omega}\left[x_{0}\right]\right\| .
$$

Notice that $\omega_{w}\left(x_{n}\right) \subset \bigcap_{n=1}^{\infty} F i x\left(S_{n}\right) \cap \Omega$. Then, (12) and Lemma 2.5 ensure the strong convergence of $\left\{x_{n+1}\right\}$ to $P_{\bigcap_{n=1}^{\infty} F i x\left(S_{n}\right) \cap \Omega}\left[x_{0}\right]$. Consequently, $\left\{y_{n}\right\}$ and $\left\{z_{n}\right\}$ also converge strongly to $P_{\bigcap_{n=1}^{\infty}}^{\infty} \operatorname{Fix}\left(S_{n}\right) \cap \Omega\left[x_{0}\right]$. This completes the proof.

\section{Acknowledgments}

The authors thank the anonymous referee for helpful comments and suggestions which improved the presentation of this paper.

Yonghong Yao was supported in part by NSFC 11071279 and NSFC 71161001G0105. Yeong-Cheng Liou was partially supported by the Taiwan NSC grant 100-2221-E-230-012.

\section{References}

[1] A.S. Antipin, Methods for solving variational inequalities with related constraints, Comput. Math. Math. Phys., 40(20007), 1239-1254. 
[2] L.C. Ceng, S. Al-Homidan, Q. H. Ansari and J.-C. Yao, An iterative scheme for equilibrium problems and fixed point problems of strict pseudocontraction mappings, J. Comput. Appl. Math., 223(2009), 967-974.

[3] L.C. Ceng, M. Teboulle and J.C. Yao, Weak convergence of an iterative method for pseudomonotone variational inequalities and fixed point problems, J. Optim. Theory Appl., 146(2010), 19-31.

[4] L.C. Ceng and J.C. Yao, Strong convergence theorem by an extragradient method for fixed point problems and variational inequality problems, Taiwan. J. Math., 10(2006), 1293-1303.

[5] L.C. Ceng and J.C. Yao, An extragradient-like approximation method for variational inequality problems and fixed point problems, Appl. Math. Comput., 1906(2007), 206-215.

[6] F. Cianciaruso, V. Colao, L. Muglia and H.K. Xu, On an implicit hierarchical fixed point approach to variational inequalities, Bull. Austral. Math. Soc., 80(2009), 117-124.

[7] F. Cianciaruso, G. Marino, L. Muglia and Y. Yao, On a two-step algorithm for hierarchical fixed Point problems and variational inequalities, J. Inequalities Appl., 2009(2009), Article ID 208692, 13 pages, doi:10.1155/2009/208692.

[8] F. Facchinei and J.S. Pang, Finite-dimensional variational inequalities and complementarity problems, Springer Series in Operations Research, vols. I and II. Springer, New York (2003).

[9] R. Glowinski, Numerical methods for nonlinear variational problems, Springer, New York, NY, 1984.

[10] K. Goebel and W. A. Kirk, Topics in Metric Fixed Point Theory, Cambridge Studies in Advanced Mathematics, vol. 28, Cambridge University Press, 1990.

[11] B.S. He, Z.H Yang and X.M. Yuan, An approximate proximalextragradient type method for monotone variational inequalities, J. Math. Anal. Appl., 300(2004), 362-374.

[12] A.N. Iusem, An iterative algorithm for the variational inequality problem, Comput. Appl. Math., 13(1994), 103-114.

[13] G.M. Korpelevich, An extragradient method for finding saddle points and for other problems, Ekonomika i Matematicheskie Metody, 12(1976), 747756 . 
[14] J.L. Lions and G. Stampacchia, Variational inequalities, Comm. Pure Appl. Math., 20(1967), 493-517.

[15] X. Lu, H. K. Xu, and X. Yin, Hybrid methods for a class of monotone variational inequalities, Nonlinear Anal., 71(2009), 1032-1041.

[16] C. Matinez-Yanes and H.K. Xu, Strong convergence of the CQ method for fixed point processes, Nonlinear Anal., 64(2006), 2400-2411.

[17] N. Nadezhkina and W. Takahashi, Strong convergence theorem by a hybrid method for nonexpansive mappings and Lipschitz-continuous monotone mappings, SIAM J. Optim., 16(2006), 1230-1241.

[18] Z. Opial, Weak convergence of the sequence of successive approximations of nonexpansive mappings, Bull. Amer. Math. Soc., 73(1967), 595-597.

[19] R.T. Rockafellar, Monotone operators and the proximal point algorithm, SIAM J. Control Optim., 14(1976),877-898.

[20] K. Shimoji and W. Takahashi, Strong convergence to common fixed points of infinite nonexpasnsive mappings and applications, Taiwanese J. Math., $5(2001), 387-404$.

[21] G. Stampacchia, Formes bilineaires coercitives sur les ensembles convexes, C.R. Acad. Sci. Paris, 258(1964), 4413-4416.

[22] W. Takahashi and M. Toyoda, Weak convergence theorems for nonexpansive mappings and monotone mappings, J. Optim. Theory Appl., 118(2003), 417-428.

[23] H.K. Xu and T. H. Kim, Convergence of hybrid steepest-descent methods for variational inequalities, J. Optimiz. Theory Appl., 119(2003), 185-201.

[24] J.C. Yao, Variational inequalities with generalized monotone operators, Math. Operations Research, 19(1994), 691-705.

[25] Y. Yao, R. Chen and H.K. Xu, Schemes for finding minimum-norm solutions of variational inequalities, Nonlinear Anal., 72(2010), 3447-3456.

[26] Y. Yao, Y.C. Liou and R. Chen, Convergence theorems for fixed point problems and variational inequality problems in Hilbert spaces, Math. Nachr., 282(12)(2009), 1827-1835.

[27] Y. Yao, Y.-C. Liou, and J.-C. Yao, Convergence theorem for equilibrium problems and fixed point problems of infinite family of nonexpansive mappings, Fixed Point Theory and Applications, 2007(2007), Article ID 64363, 12 pages. 
[28] Y. Yao and M.A. Noor, On viscosity iterative methods for variational inequalities, J. Math. Anal. Appl., 325(2007), 776-787.

[29] Y. Yao and M.A. Noor, On modified hybrid steepest-descent methods for general variational inequalities, J. Math. Anal. Appl., 334(2007), 12761289.

[30] Y. Yao and M.A. Noor, On modified hybrid steepest-descent method for variational inequalities, Carpathian J. of Math., 24(2008), 139-148.

[31] Y, Yao and J.C. Yao, On modified iterative method for nonexpansive mappings and monotone mappings, Appl. Math. Comput., 186(2007), 15511558 .

$\mathrm{Xin} \mathrm{Yu}$,

School of Science, Tianjin Polytechnic University,

Tianjin 300387, China.

Email: lxy@tjpu.edu.cn

Yonghong Yao,

Department of Mathematics, Tianjin Polytechnic University,

Tianjin 300387, China.

Email: yaoyonghong@yahoo.cn

Yeong-Cheng Liou,

Department of Information Management, Cheng Shiu University,

Kaohsiung 883, Taiwan.

Email: simplex_liou@hotmail.com 
